\title{
Shielding calculations for industrial 5/7.5MeV electron accelerators using the MCNP Monte Carlo Code
}

\author{
Eyal Peri ${ }^{1, *}$ and Itzhak Orion ${ }^{2}$ \\ ${ }^{1}$ Nuclear Research Centre Negev, P.O.B 9001, Beer-Sheva, 84190, Israel \\ ${ }^{2}$ Ben-Gurion University of the Negev, Beer Sheva, Israel
}

\begin{abstract}
High energy X-rays from accelerators are used to irradiate food ingredients to prevent growth and development of unwanted biological organisms in food, and by that extend the shelf life of the products. The production of X-rays is done by accelerating $5 \mathrm{MeV}$ electrons and bombarding them into a heavy target (high Z). Since 2004, the FDA has approved using 7.5 MeV energy, providing higher production rates with lower treatments costs. In this study we calculated all the essential data needed for a straightforward concrete shielding design of typical food accelerator rooms. The following evaluation is done using the MCNP Monte Carlo code system: (1) Angular dependence $\left(0-180^{\circ}\right)$ of photon dose rate for $5 \mathrm{MeV}$ and 7.5 $\mathrm{MeV}$ electron beams bombarding iron, aluminum, gold, tantalum, and tungsten targets. (2) Angular dependence $\left(0-180^{\circ}\right)$ spectral distribution simulations of bremsstrahlung for gold, tantalum, and tungsten bombarded by $5 \mathrm{MeV}$ and $7.5 \mathrm{MeV}$ electron beams. (3) Concrete attenuation calculations in several photon emission angles for the $5 \mathrm{MeV}$ and $7.5 \mathrm{MeV}$ electron beams bombarding a tantalum target. Based on the simulation, we calculated the expected increase in dose rate for facilities intending to increase the energy from $5 \mathrm{MeV}$ to $7.5 \mathrm{MeV}$, and the concrete width needed to be added in order to keep the existing dose rate unchanged.
\end{abstract}

\section{Introduction}

Industrial electron accelerators are used for many applications such as: irradiation of medical devices, food and wires, etc. [1]. In this study we calculated essential data needed for a straightforward concrete shielding design of an industrial electron accelerator, using $5 \mathrm{MeV}$ and 7.5 MeV X-Rays. These energies are mainly used to irradiate food ingredients. Food irradiation using ionizing radiation is a common process to prevent the growth and development of several types of biological organisms in food. In the past, the main process to provide the desired dose required a ${ }^{60} \mathrm{Co}$ irradiation facility. Nowadays, due to their inherent safety, electron accelerators are more in use. When deeper penetration is needed, the electron beam produces bremsstrahlung (Xrays) by using targets with high $Z$, acting as convertors.

Due to the low range of electrons in matter, irradiation by the electron beam is limited to small packages. To irradiate larger packages, higher penetration is needed, therefore the electron beam has to be converted to bremsstrahlung X-rays. The targets used to convert the electron beam to bremsstrahlung are tantalum, tungsten, gold and aluminum. Aluminum is used as an electron beam absorber.

High energy photons can cause food activation due to $(\gamma, \mathrm{n})$ reactions. Until 2004 , to eliminate the possibility of food activation, the electron energy was limited to 5 $\mathrm{MeV} \mathrm{X}$-rays for food irradiation. In 2004, the FDA approved the usage of up to $7.5 \mathrm{MeV}$, but only with tantalum and gold targets [2].

Higher X-ray energy results an increased flux of Xrays in the forward direction, increased penetration, and higher photon dose rate due to better electron-to-photon conversion. These improvements could decrease the irradiation time and allow irradiation of larger packages, thereby providing higher production rates with lower treatment cost.

Electron accelerators emit high currents of high energy electrons and X-Ray. The X-ray energies are determined by the beam energy; the higher the energy, the better the X-ray penetration. The magnitude of the accelerator dose rates is mainly a function of the beam current. The typical dose rates in medical accelerator are quite low, up to $100 \mathrm{~Gy} \mathrm{~h}^{-1}$, compared to the dose rates in industrial accelerators, which are much higher: 100 $\mathrm{kGy} \mathrm{h}^{-1}$ in X-Ray mode, and up to $360,000 \mathrm{kGy} \mathrm{h}^{-1}$ in electron irradiation mode [1]. Due to the difference in dose rates, the shielding thickness of industrial accelerator is much higher. Therefore, accurate shielding values such as angular dependence of dose rates and concrete attenuation ratios, is very important for designing the optimal shielding.

Medical accelerators usually work with 6-18 MV electron energy with a tungsten target to convert the electron beam to X-rays. In order to protect the patients, the accelerator head is protected with a heavy lead shielding; therefore, the bremsstrahlung radiation is emitted only in the forward direction. Shielding thickness calculations are based on available bremsstrahlung spectrum data in the forward direction (primary shielding). Secondary shielding is designed to protect against photon scattering from the primary shielding or leakage from the accelerator head, which are two orders of magnitude smaller than the primary beam [3]. There are many publications and standards that guide how to design optimal shielding for medical accelerator rooms [3-5]. The shielding data for medical

* Corresponding author: eyalperi22@gmail.com 
accelerators is not applicable for industrial accelerators, since the data is for different conversion targets, different X-Ray energies, and only for the forward direction.

In general, collimators are not in use in industrial accelerators, since human protection is not needed inside the room (e.g. patients), and therefore bremsstrahlung photons can be emitted in all directions. The bremsstrahlung spectrum and dose rate change as a function of the emission angle. The dose rate decreases from maximum in the forward direction $\left(0^{\circ}\right)$ to minimum at $180^{\circ}$ by $1-2$ orders of magnitude. In order to design and calculate optimal shielding for food accelerator rooms, there is a need to have the bremsstrahlung spectrum data and dose rates in all emission directions, which are different for each conversion target and energy (5 $\mathrm{MeV}$ or $7.5 \mathrm{MeV}$ ). There are publications and standards available such as: NCRP 144 [6], IAEA Report 188 [7], ANSI N43.3 [8], to guide how to design shielding for industrial accelerator rooms. The shielding data in these guides is suitable for most industrial accelerators, but does not contain data for $5 \mathrm{MeV}$ and 7.5 $\mathrm{MeV}$ X-rays, such as used in food irradiation facilities.

One publication written specifically to give data for the shielding design of food accelerator rooms was published by Barkova [9]. The angular dependence of the KERMA rates from aluminum, iron, and tungsten targets irradiated by $5 \mathrm{MeV}$ electrons were calculated using Monte Carlo simulations with the use of the program package SPIN-PC, presented by Barkova [9]. The concrete attenuation ratio was evaluated analytically using Monte Carlo simulations to obtain the bremsstrahlung spectrum for each angle.

Petwal [10] performed MCNP calculations to optimize the design of the conversion target for maximizing the X-ray output. They also presented calculated $5 \mathrm{MeV}$ and $7.5 \mathrm{MeV}$ relative bremsstrahlung spectra, but only for the forward direction, and relative angular dose rate up to $60^{\circ}$. The authors point out that from the three possible targets (gold, tantalum, tungsten), tantalum is the most suitable for reasons of photon yield, residual radioactivity, mechanical properties, and fabrication simplicity.

\section{Objective}

The following evaluations were done using the MCNP Monte Carlo code system. Simulations for angular dependence calculations of photon dose rate in units of Gy $\mathrm{h}^{-1}$ were carried out at the distance of one meter from several common converting targets as a function of the beam current for $5 \mathrm{MeV}$ and $7.5 \mathrm{MeV}$ electron beams. Photon attenuation ratios in concrete were calculated for a range of $0-180 \mathrm{~cm}$ for a tantalum target at selected photon emission angles $\left(0^{\circ}, 90^{\circ}, 135^{\circ}, 180^{\circ}\right)$ for the 5 $\mathrm{MeV}$ and the $7.5 \mathrm{MeV}$ electron beams. Based on the simulation, we calculated the expected increase in dose rate for facilities intending to increase the energy from 5 $\mathrm{MeV}$ to $7.5 \mathrm{MeV}$.

\section{Materials and methods}

The simulations were carried out using the MCNP version 4C2 code package [11]. Initiation of MCNP requires an input file that contains complete information about the radiation source, the geometry of the system to be simulated, and both the density and the elemental composition of all interacting materials. The Concrete composition was taken as an ordinary concrete $2.3 \mathrm{~g} \mathrm{~cm}^{-}$ 3 in density [12].

In addition, the output tallies to be calculated need to be specified in the MCNP input file. The tally F6 in a cell volume was used for absorbed dose calculations. The dose was calculated by employing the MCNP 'F6:p' track length tally and making the KERMA approximation. Since most of the dose is by photons of less than $3 \mathrm{MeV}$, electron equilibrium is satisfied, than the terms 'dose' and 'KERMA' are thus used synonymously in this work. Tally *F4 of photon fluence was used for the photon spectra graphs.

Simulation of the angular dependence of photon dose rate $\left(\mathrm{Gy} \mathrm{h}^{-1}\right)$ at one meter from the targets as a function of beam current $(\mathrm{mA})$ and beam energy were done by irradiating gold, tantalum, and tungsten targets with a 5/7.5 MeV pencil electron beam. In real accelerators the beam dimension is not narrow, but the distance from the source to the exterior side of the building is more than a few meters. Therefore, for shielding purposes it is possible to simulate the electron beam as a narrow beam to simplify the simulation. The targets depth and radial dimensions were chosen to be about 20\% less the CSDA range using ESTAR database [13], which is approximately $20 \%$ more than the penetration range of the electrons in the target [14]. Target thickness of $\mathrm{Al}, \mathrm{Fe}, \mathrm{Ta}, \mathrm{W}$ and $\mathrm{Au}$ were 9.4, 3.2, $1.5,1.3$ and $1.3 \mathrm{~mm}$ for $5 \mathrm{MeV}$ electrons, and target thickness of $\mathrm{Ta}$ and $\mathrm{Au}$ were 2.3 and $2.0 \mathrm{~mm}$ for 7.5 $\mathrm{MeV}$ electrons.

The three-dimensional space was subdivided into conical shells to 18 sectors, from $0^{\circ}$ (forward direction) to $180^{\circ}$ (backward), $10^{\circ}$ for each sector. At a radius of 1 meter around the target, 18 volumes of "detectors" were made by generating two concentric spheres at $99 \mathrm{~cm}$ and $101 \mathrm{~cm}$, and dividing them by the 18 sectors. All the space around the target was covered by 18 cells, defined as "detectors" filled with standard air. The bremsstrahlung photons were collected by the 18 "detectors". The results were converted to units of (Gy $\mathrm{mA}^{-1} \mathrm{~h}^{-1}$ ) at a distance of $1 \mathrm{~m}$ from the target, for each $10^{\circ}$ sector. Since every photon was collected by one of the $10^{\circ}$ "detectors", the number of photons reaching each detector was large. Therefore the statistics was adequate, with a small standard deviation (less than 2\%) obtained with very short run time (e.g., 1 hour). For shielding purposes, averaging the dose rate at a $10^{\circ}$ angle does not consider significant error. In order to validate the correctness of the results, simulations of aluminum, iron, and tungsten were done and compared to the data of Barkova [9]. As can be seen in Figure 1, the results are in good agreement, except in aluminum and iron targets, 
in the forward directions the difference that our results are higher up to $20 \%$ than the previous study results.

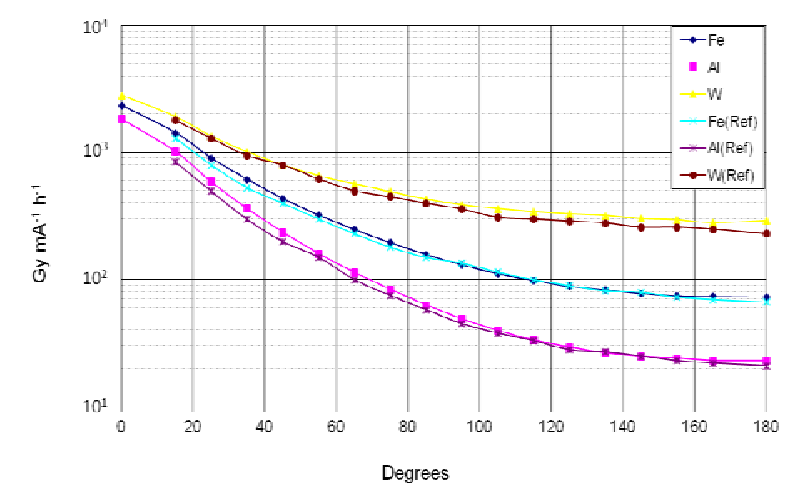

Fig. 1. Angular dependence of photon dose rate $\left(\mathrm{Gy} \mathrm{h}^{-1}\right)$ at one meter from $\mathrm{Fe}, \mathrm{Al}, \mathrm{W}$ targets as a function of beam current (mA) for $5 \mathrm{MeV}$ electron beam. MCNP (our result) vs. Barkova [9].

Simulation of the energy distribution of bremsstrahlung emission for gold, tantalum, and tungsten targets by $5 \mathrm{MeV}$ and $7.5 \mathrm{MeV}$ electron beams were performed. Energy distribution of bremsstrahlung intensity was calculated using Tally ${ }^{*} \mathrm{~F} 4$ as $\mathrm{kdn} \mathrm{dk}^{-1} \mathrm{~d} \Omega^{-1}$ $\left(\mathrm{MeV} \mathrm{MeV}^{-1} \mathrm{Sr}^{-1}\right.$ electron $\left.{ }^{-1}\right)$, by dividing each bin by its energy interval and multipling it by the square of the distance. The plots are presented as the number of bremsstrahlung photons of energy $\mathrm{k}$ within an increment of energy $\mathrm{dk}$, emitted at angle $\theta$ within a solid angle $\mathrm{d} \Omega$, by one electron whose initial energy is $E_{0}$. The same geometry configuration like the previous section was provided. The bremsstrahlung photons were collected in the "detectors" cells. The energy bins were $0.2 \mathrm{MeV}$ at the following angles: $0^{\circ}, 10^{\circ}, 20^{\circ}, 45^{\circ}, 60^{\circ}, 90^{\circ}, 120^{\circ}$, $150^{\circ}$, and $180^{\circ}$. Each angle is the average of $\pm 5^{\circ}$ around it. In order to validate the results, the first simulation was compared to the spectrum of $2.8 \mathrm{MeV}$ electrons hitting an iron target as measured by Dance [15]. Three emission angles were selected for comparison $\left(10^{\circ}, 45^{\circ}\right.$, $120^{\circ}$ ); as can be seen in Figure 2, the results showed a good agreement.

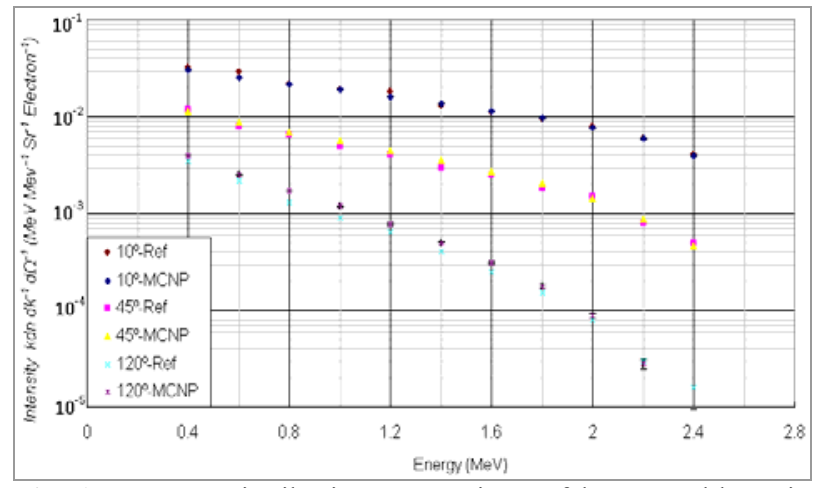

Fig. 2. Energy Distribution comparison of bremsstrahlung in Iron target $2.8 \mathrm{MeV}$. MCNP (our result) vs. Fig. 11 in Dance [15].

Simulation of photon attenuation in concrete of selected photon emission angles $\left(0^{\circ}, 90^{\circ}, 135^{\circ}\right.$, and $180^{\circ}$ ) were performed for $5 \mathrm{MeV}$ and $7.5 \mathrm{MeV}$ electron beams. The same configurations as the previous section were done. A spherical concentric concrete shield was added between the target and detectors from a thickness of $10 \mathrm{~cm}$ up to $180 \mathrm{~cm}$, hence the detectors were moved to $200 \mathrm{~cm}$. Every $10 \mathrm{~cm}$ addition required a separated run; when simulating concrete shielding thicker than 80 $\mathrm{cm}$, only a few photons were tallied and there was a need to use variance reduction techniques in order to get improved statistics. The attenuation data were calculated for four emission angles. In order to improve the statistics, broad angles were chosen (up to $\pm 20^{\circ}$ ). Since there is an increased concentration of bremsstrahlung photons in the forward direction, the collection angle was set to be larger in the backward direction than in the forward direction. The emission angles used were: $0^{\circ}(0$ $\left.10^{\circ}\right), 90^{\circ}\left(80-100^{\circ}\right), 135^{\circ}\left(120-150^{\circ}\right)$, and $180^{\circ}\left(160^{\circ}\right.$ $180^{\circ}$ ) (Figure 3). For shielding purposes, averaging the backward radiation dose rate in $\mathrm{a} \pm 20^{\circ}$ range does not lead to significant uncertainties. The variance reduction technique that was used is geometry splitting: As illustrated in Figure 3, the concrete was divided to $10 \mathrm{~cm}$ sections. The importance of each section was gradually increased from the inner to the outer sections, and the importance of the backwards direction was greater than in the forward direction, so the photon population will be approximately equal. Figure 3 is a cross-section illustration of the geometry, and illustrates a single run with $80 \mathrm{~cm}$ of concrete.

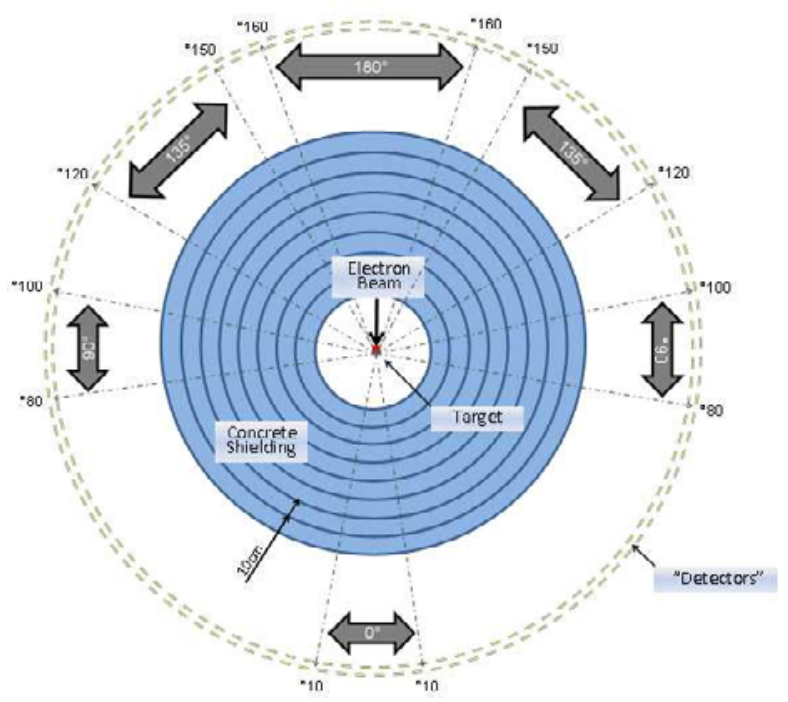

Fig. 3. Illustration of concrete shielding between the target and the detectors at $0^{\circ}, 90^{\circ}, 135^{\circ}$, and $180^{\circ}$.

In order to validate the results, the attenuation simulations results of an aluminium target with emission angles of $90^{\circ}$ and $180^{\circ}$ were compared to the data published in Barkova [9]. As can be seen in Figure 4, the results showed a good agreement. 


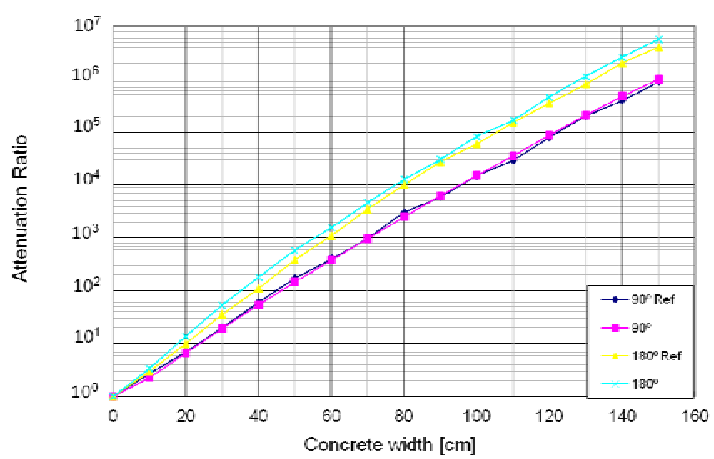

Fig. 4. Dose attenuation by concrete of the bremsstrahlung emission from $\mathrm{Al}$ target for beam energy of $5 \mathrm{MeV}$. MCNP (this study) vs. Barkova [9].

\section{Results}

Figure 5 presents the angular dependence $\left(0-180^{\circ}\right)$ of photon dose rate $\left(\mathrm{Gy} \mathrm{h}^{-1}\right)$ at $1 \mathrm{~m}$ from gold, tantalum, and tungsten targets as a function of beam current (mA) for $5 \mathrm{MeV}$ and $7.5 \mathrm{MeV}$ electron beams. 106 $-10^{7}$ histories were used to obtain relative statistical uncertainties lower than 2\%. Error bars for less than 5\% are too small to be shown in the graphs. The results of tantalum and tungsten for $5 \mathrm{MeV}$ are almost identical. The reason is the close atomic number of the two targets. Due to the larger atomic number of gold its dose rate is slightly higher, but only for the backward angles $\left(>90^{\circ}\right)$. This is one of the reasons why a gold target is not cost effective. The $7.5 \mathrm{MeV}$ dose rates curves show almost the same shape of the ones relating to $5 \mathrm{MeV}$ and are higher by a factor from 1.5 up to 2.6.

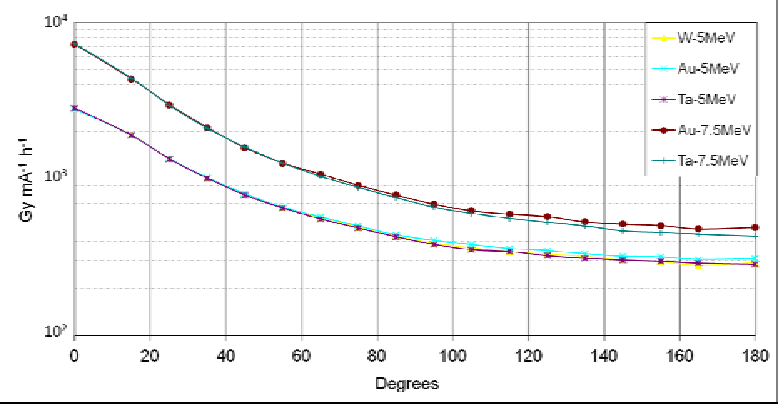

Fig. 5. Angular dependence of photon dose rate $\left(\mathrm{Gy} \mathrm{h}^{-1}\right)$ at one meter from $\mathrm{Au}, \mathrm{Ta}, \mathrm{W}$ targets as a function of beam current (mA) for $5 / 7.5 \mathrm{MeV}$ electron beam.

Figures 6 and 7 present the energy distribution of bremsstrahlung emission for gold and tantalum targets by $5 \mathrm{MeV}$ and $7.5 \mathrm{MeV}$ electron beams. $10^{7}-10^{8}$ histories were used to usually obtain relative uncertainties lower than $5 \%$. Due to the small difference in the atomic numbers of the targets, the spectra are almost identical for each energy. The spectrum of the forward direction photons is higher and "harder" than the spectrum in the backward emission. In the lower energy bins, the fluence in the forward directions is higher by an order of magnitude than the backward emission. As the energy bins increased, the gap increases up to three orders of magnitudes in the higher energy bins. From these figures we can understand why the dose rate is decreasing as the photon emission angle increases. The fact the spectrum of the backward is softer explains why the concrete shielding is more effective to attenuate backward photon emission.
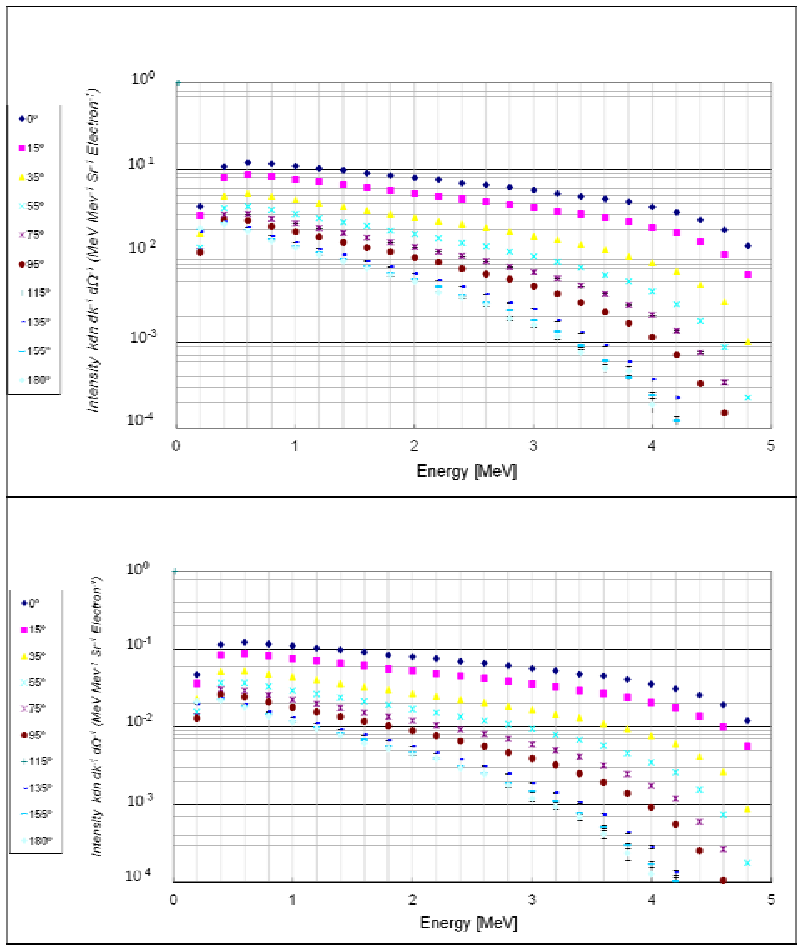

Fig. 6. Energy distribution of $5 \mathrm{MeV}$ bremsstrahlung in $\mathrm{Au}$ (Upper) and Ta (Lower) Targets.
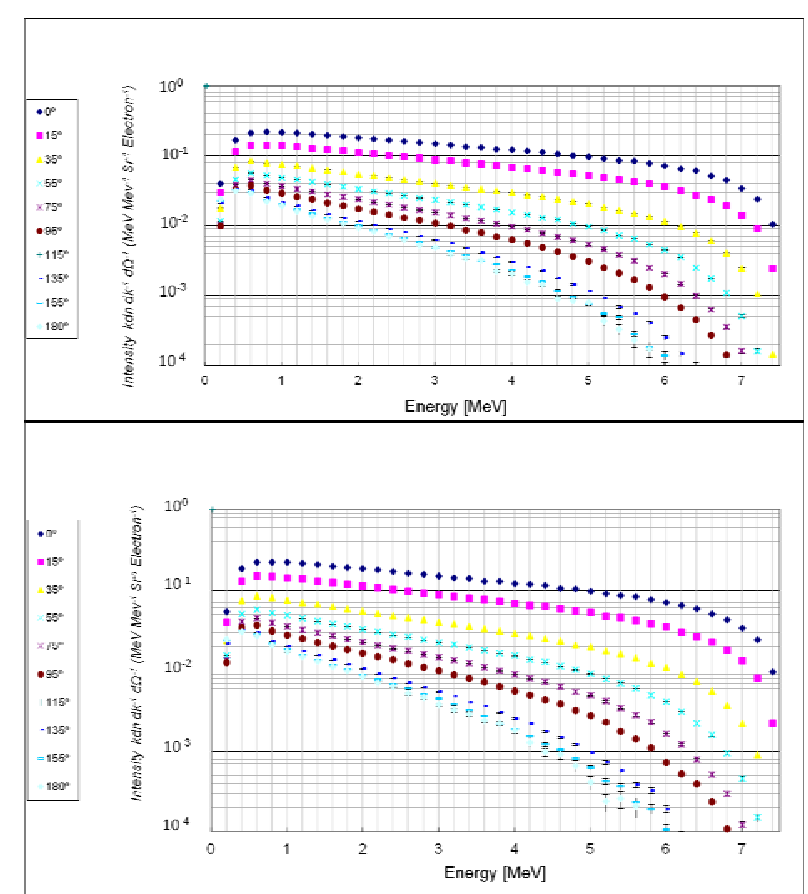

Fig. 7. Energy distribution of $7.5 \mathrm{MeV}$ bremsstrahlung in $\mathrm{Au}$ (Upper) and Ta (Lower) Targets.

Figure 8 presents the photon attenuation ratio in concrete $(0-180 \mathrm{~cm})$ of a tantalum target in selected photon emission angles $\left(0^{\circ}, 90^{\circ}, 135^{\circ}, 180^{\circ}\right)$ for $5 \mathrm{MeV}$ 
and $7.5 \mathrm{MeV}$ electron beams. The attenuation ratio was obtained as the ratio between the dose without shielding to the dose behind the concrete shielding. $10^{6}-10^{8}$ histories were used to obtain relative uncertainty less than $5 \%$. As with the dose rate and bremsstrahlung results, the data for gold and tungsten should be similar, and therefore were not calculated here. The photon attenuation in concrete for $5 \mathrm{MeV}$ is higher than for 7.5 $\mathrm{MeV}$ by up to an order of magnitude. The attenuation is larger for the backward emitted photons compared to the forward ones by up to an order of magnitude.
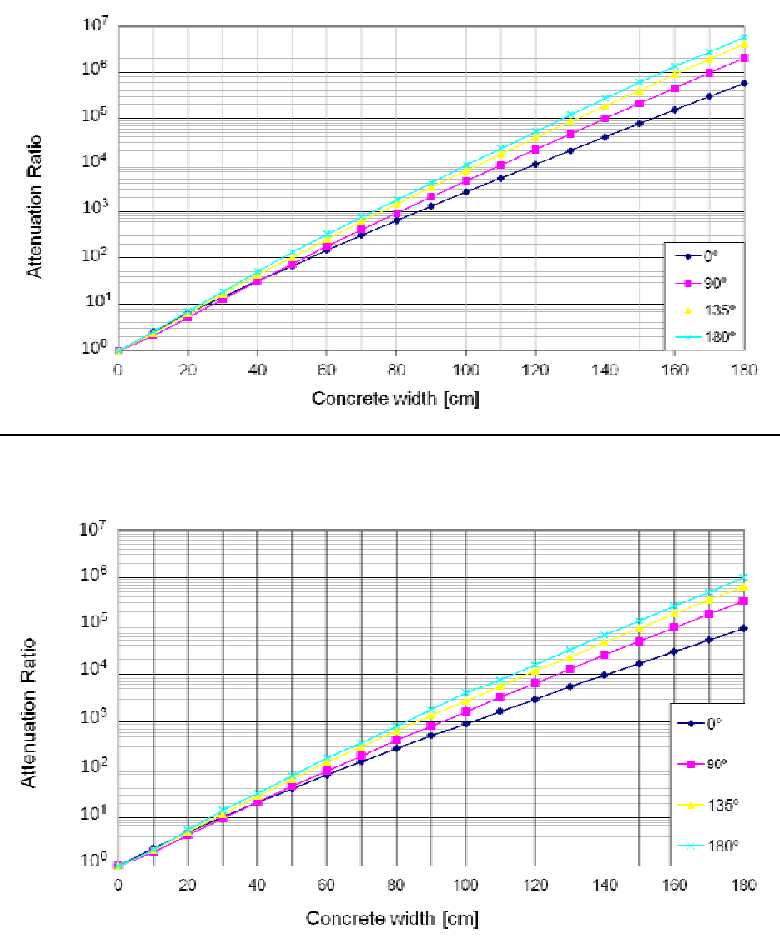

Fig. 8. Dose attenuation in concrete of the bremsstrahlung emission from $\mathrm{Ta}$ target for electron beam energy of $5 \mathrm{MeV}$ (Upper) and 7.5 MeV (Lower).

Table 1 is an example of how to calculate the expected dose rate behind concrete shielding using the graphs given in this study. The example was set for an accelerator of $1 \mathrm{~mA}$ current and a tantalum target. The listed energy, emission angle, and concrete width and distance from the target are reasonably chosen settings. The dose rate at $1 \mathrm{~m}$ from the target is taken from Figure 5 , and the attenuation ratio is taken from Figure 8; both are based on the settings data given in Table 1. The final dose rate behind the shielding at distance $\mathrm{L}$ from the target is obtained by the following equation:

$$
\dot{D_{\text {out }}}\left[\frac{\mu G y}{m A \cdot h}\right]=\frac{\dot{D}_{\text {in }}\left[\frac{G y}{m A \cdot h}\right] \cdot 10^{6}}{\left(\text { Atten.Ratio }_{\text {concrate }}\right)}\left(\frac{1[m]}{L[m]}\right)^{2}
$$$$
\dot{D}_{\text {in }} \text { - The dose rate one meter from the conversion }
$$
target; $10^{6}$ is a factor from Gy to $\mu \mathrm{Gy}$.
Table 1. Example of shielding calculation for $1 \mathrm{~mA}$ current with Ta target.

\begin{tabular}{|c|c|c|c|c|c|c|c|}
\hline Point & $\begin{array}{l}\text { Energy } \\
\text { [MeV] }\end{array}$ & $\begin{array}{c}\text { Emission } \\
\text { Angle } \\
\text { [deg.] }\end{array}$ & $\begin{array}{l}\text { Concrete } \\
\text { width } \\
{[\mathrm{cm}]}\end{array}$ & $\begin{array}{c}\text { Distance } \\
\text { [m] }\end{array}$ & $\begin{array}{c}\text { Dose Rate } \\
{\left[G y \cdot \mathrm{mA}^{-1} \cdot \mathrm{h}^{-1}\right]}\end{array}$ & $\begin{array}{c}\text { Concrete } \\
\text { Attenuation } \\
\text { ratio }\end{array}$ & $\begin{array}{c}\text { Dose Rate Behind } \\
\text { Shieding } \\
{\left[\mu C y \cdot \omega^{-1}\right]}\end{array}$ \\
\hline 1 & 5 & 90 & 170 & 7 & 400 & $1.010^{6}$ & 8 \\
\hline 2 & 5 & 180 & 120 & 8 & 285 & $5.310^{4}$ & 84 \\
\hline 3 & 7.5 & 135 & 150 & 7 & 500 & $8.810^{4}$ & 116 \\
\hline 4 & 7.5 & 90 & 160 & 11 & 700 & $9.210^{4}$ & 53 \\
\hline
\end{tabular}

${ }^{2}$ From Figure 5 (before shielding).

${ }^{3}$ From Figure 8 .

The quantities of concrete required to be added in a case of upgrading a $5 \mathrm{MeV}$ beam to $7.5 \mathrm{MeV}$ in a facility, in order to keep the $5 \mathrm{MeV}$ dose rate are listed in Table 2. The expected difference in dose rate between 5 $\mathrm{MeV}$ and $7.5 \mathrm{MeV}$ emitted from a tantalum target at $0^{\circ}$, $90^{\circ}$, and $180^{\circ}$ are presented in Table 2 . The presented results are taken from Figure $5(2.6,1.7,1.5$, respectively), multiplied by the difference in the concrete attenuation, taken from Figure 8 . The data are presented for three emission angles $\left(0^{\circ}, 90^{\circ}, 180^{\circ}\right)$ as a function of the existing concrete thickness in the facility. The data are not dependent on the distance between the target and the interest point outside the facility.

Table 2. Dose rate ratios of bremsstrahlung emission between $5 \mathrm{MeV}$ and $7.5 \mathrm{MeV}$ beams Ta target as a function of existing concrete for a given angle (left), and the quantity of required concrete to be added for $7.5 \mathrm{MeV}$ facility in order to keep the 5 $\mathrm{MeV}$ dose rate (right).

\begin{tabular}{|c|c|c|c|c|c|c|}
\hline $\begin{array}{c}\text { Existed } \\
\begin{array}{c}\text { Concrete } \\
\text { (cm) }\end{array}\end{array}$ & \multicolumn{2}{|c|}{$\begin{array}{c}\mathbf{5} \text { MeV and 7.5 MeV } \\
\text { Dose Rate Ratio }\end{array}$} & \multicolumn{3}{|c|}{$\begin{array}{c}\text { Required Concrete } \\
\text { addition (cm) }\end{array}$} \\
\hline & $\mathbf{0}^{\circ}$ & $\mathbf{9 0}^{\circ}$ & $\mathbf{1 8 0 ^ { \circ }}$ & $\mathbf{0}^{\circ}$ & $\mathbf{9 0}^{\circ}$ & $\mathbf{1 8 0}^{\circ}$ \\
\hline 0 & 2.6 & 1.7 & 1.5 & 8 & 5 & 0.5 \\
\hline 10 & 2.8 & 1.9 & 1.6 & 9 & 6 & 2 \\
\hline 20 & 3.3 & 2.0 & 1.7 & 12 & 7 & 2 \\
\hline 30 & 3.5 & 2.3 & 1.8 & 13 & 9 & 3 \\
\hline 40 & 4.0 & 2.5 & 2.0 & 15 & 10 & 4 \\
\hline 50 & 4.2 & 2.8 & 2.1 & 16 & 12 & 5 \\
\hline 60 & 4.8 & 3.3 & 2.2 & 18 & 14 & 5 \\
\hline 70 & 5.4 & 3.6 & 2.5 & 19 & 15 & 7 \\
\hline 80 & 5.9 & 3.8 & 2.7 & 21 & 16 & 8 \\
\hline 90 & 6.5 & 4.3 & 2.8 & 23 & 18 & 9 \\
\hline 100 & 7.6 & 4.9 & 2.9 & 25 & 20 & 9 \\
\hline 110 & 8.2 & 5.1 & 3.5 & 26 & 20 & 12 \\
\hline 120 & 9.0 & 6.0 & 3.8 & 28 & 22 & 13 \\
\hline 130 & 9.7 & 6.3 & 4.1 & 29 & 23 & 14 \\
\hline 140 & 11.1 & 6.9 & 4.4 & 31 & 24 & 15 \\
\hline 150 & 12.2 & 7.6 & 4.9 & 33 & 26 & 16 \\
\hline 160 & 13.7 & 8.6 & 5.2 & 35 & 28 & 17 \\
\hline 170 & 15.3 & 9.4 & 5.8 & 36 & 29 & 18 \\
\hline 180 & 16.5 & 10.5 & 6.1 & 38 & 31 & 19 \\
\hline & & & & & & \\
\hline
\end{tabular}

By using Table 2, facilities that plan upgrading their accelerator to $7.5 \mathrm{MeV}$ can easily estimate the expected increase in dose rate around the shielded facility. The additional concrete thickness ensuring the same dose rate around the facilty can also be evaluated, thus maintaining the same safety status. For example, a 
facility interested in the dose rate over a $120 \mathrm{~cm}$ shield located $90^{\circ}$ from the electron beam direction, can use Table 2 to find that the expected dose rate rises by a factor of 6 , or an additional $22 \mathrm{~cm}$ of concrete thickness to that area should lower the dose rate back to the original rate.

\section{Conclusions}

Angular dependence MCNP simulations of dose rate, bremsstrahlung, and concrete attenuation ratio were carried out for $5 \mathrm{MeV}$ and $7.5 \mathrm{MeV}$ accelerators for gold, tantalum, and tungsten targets. Since the targets atomic numbers are similar, there was only a slight difference in dose rates and bremsstrahlung spectra of the targets. The dose rate of $7.5 \mathrm{MeV}$ accelerators is higher than the $5 \mathrm{MeV}$ dose rate by factor of about 2.6 in the forward direction, and a factor of 1.5 in the backward direction. The difference in dose rates from the forward to backward directions is almost two orders of magnitude. Photon attenuation ratios in concrete of 7.5 $\mathrm{MeV}$ beam is lower than that of $5 \mathrm{MeV}$ beam by almost an order of magnitude for thick shielding for all emission angles. There is a significant difference in the bremsstrahlung spectrum between the forward emission angles to the backward angles. Since the bremsstrahlung spectrum of the backward angles is softer than in the forward direction, the concrete shielding is more effective in attenuating the backward direction. This difference is small for thin shielding, but for thick shielding of more than $150 \mathrm{~cm}$, the attenuation of the backward emissions is larger than for forward direction by an order of magnitude, for the same concrete thickness.

\section{References}

1. IAEA, Industrial Radiation Processing with Electron Beam and X-Rays - Revision 6, 1 May 2011.

2. Federal Register. 69(246):76844; 2004.

3. IAEA, Safety Report Series No.47, Radiation Protection in the design of Radiotherapy Facilities, 2006.

4. NCRP Report No.49, Structural Shielding Design and Evaluation for Medical Use of X-Rays and Gamma-Rays up to $10 \mathrm{MeV}, 1976$.

5. NCRP Report No.151, Structural Shielding Design and Evaluation for Megavoltage X and Gamma Ray Radiotherapy Facilities, 2005.

6. NCRP Report No.144. Radiation Protection for Particle Accelerator Facilities; 2005.

7. IAEA, Technical Reports Series No.188, Radiological Safety Aspects of the Operation of Electron Linear Accelerators 1979.

8. ANSI/HPS N43.3-2008, For General Radiation Safety - Installation Using Non-Medical X-ray and Sealed Gamma-Ray Sources, Energy Up to $10 \mathrm{MeV}$, 2008.

9. Barkova VG, Kiselev AV, Chudaev VYa. Evaluation of Efficiency of Concrete Shielding Against Bremmsstrahlung of $5 \mathrm{Mev}$ Electrons at
Pre-Commissioning of the Accelerator ILU-10, Proceeding of RuPAC, Novosibirsk, Russia; 2006.

10. Petwal VC, Bapna SC, Sanda RS, Kotaiah S, Subbaih KV. Bremsstrahlung Converter for High Power EB Radiation Processing Facility, APAC, RRCAT. Indore, India; 2007.

11. Briesmeister JF, ed. MCNP A General Monte Carlo N-Particle Transport Code, version 4C, LA-13709M. Los Alamos National Laboratory, Los Alamos, NM; April 2000.

12. Williams R.G., Compendium of Material Composition Data for Radiation Transport Modeling, DOE, April 2006.

13. http://physics.nist.gov/PhysRefData/Star/Text/EST AR.html

14. Attix F H, Introduction to Radiological Physics and Radiation Dosimetry, pp. 184-185, Wiley 2004.

15. Dance WE, Rester DH, Farmer BJ, Johonson JH. Bremsstrahlung Produced in Thick Aluminum and Iron Targets by 0.5 to $2.8 \mathrm{MeV}$ Electrons. J Appl Phys 39(6):2881-2889; $1967 . \quad$ DOI: http://dx.doi.org/+10.1063/1.1656689 